\title{
FORMAS DE ADUBAÇÃO E DOSES DE URÉIA PARA MUDAS CLONAIS DE CACAU CULTIVADAS EM SUBSTRATO ${ }^{(1)}$
}

\author{
José Olimpio de Souza Júnior ${ }^{(2)}$ \& Quirino Augusto de Camargo Carmello ${ }^{(3)}$
}

\begin{abstract}
RESUMO
O N é o nutriente mais demandado pelas plantas. Contudo, para produção de mudas enraizadas de cacaueiro em substratos e sob irrigação freqüente não existem estudos de calibração de adubação nitrogenada. Os objetivos deste trabalho foram comparar a eficiência das adubações no substrato e foliar com uréia para mudas clonais de cacau e definir doses recomendáveis e nível crítico foliar de N. O experimento foi instalado em viveiro de produção de mudas do Instituto Biofábrica de Cacau, em Ilhéus, Bahia, em julho de 2006, envolvendo 10 tratamentos (duas formas de adubação, combinadas com cinco doses de uréia), em blocos casualizados, com quatro repetições. A parcela útil constituiu-se de 12 mudas clonais de cacaueiro cultivadas em tubetes, contendo $288 \mathrm{~cm}^{3} \mathrm{de}$ substrato (50\% de fibra de coco e $50 \%$ de Plantmax ${ }^{\circledR}$ ). O substrato foi corrigido com $3,20 \mathrm{~kg} \mathrm{~m}^{-3}$ de calcário e adubado com $2,68 \mathrm{~kg} \mathrm{~m}^{-3}$ de superfosfato simples. Os tratamentos foram aplicados entre o $82^{\circ}$ até o $138^{\circ}$ dia, com as seguintes doses semanais de $\mathrm{N}$ : 0, 20, 40, 60 e $80 \mathrm{mg} \mathrm{dm}^{-3}$ via substrato e 0,$0 ; 2,25 ; 4,5 ; 9,0 ;$ e 13,5 $\mathrm{g} \mathrm{L}^{-1}$ via foliar. Em todos os tratamentos, fez-se a adubação complementar com P, K, S, Zn e B via substrato. Aos 145 dias, as plantas foram colhidas, sendo avaliados: diâmetro da haste principal, altura, área foliar, matéria seca da parte aérea (MSPA), teor de nutrientes na folha diagnóstica e conteúdo de nutrientes na parte aérea. As variáveis biométricas e nutricionais responderam aos tratamentos. A adubação via substrato proporcionou produção máxima superior à adubação foliar, para MSPA, altura e área foliar. As doses recomendáveis de N, calculadas para obtenção de 99 \% da produção máxima, variaram de acordo com a variável biométrica de 43 a $76 \mathrm{mg} \mathrm{dm}^{-3}$ para adubação via substrato e de 9,1 a $11,7 \mathrm{~g} \mathrm{~L}^{-1}$ para adubação foliar. O nível crítico foliar de $\mathrm{N}$, para obtenção de 99 \% da produção máxima da MSPA, foi de $23,1 \mathrm{~g} \mathrm{~kg}^{-1}$.
\end{abstract}

Termos de indexação: Theobroma cacao, nutrição, adubação foliar, nível crítico, cultivo sem solo, nitrogênio.

\footnotetext{
(1) Recebido para publicação em novembro de 2007 e aprovado em setembro de 2008 .

(2) Professor do Departamento de Ciências Agrárias e Ambientais, Universidade Estadual de Santa Cruz - UESC. km 16, Rod. Ilhéus/Itabuna, CEP 45652-000 Ilhéus (BA). E-mail: olimpio@uesc.br

(3) Professor do Departamento de Ciência do Solo, Escola Superior de Agricultura "Luiz de Queiroz", Universidade de São Paulo - ESALQ/USP. Av. Pádua Dias 11, Caixa Postal 09, CEP 13418-900 Piracicaba (SP). E-mail: qaccarme@esalq.usp.br
} 


\title{
SUMMARY: FORMS AND DOSES OF UREA TO FERTILIZE CLONAL COCOA TREE CUTTINGS CULTIVATED IN SUBSTRATE
}

\begin{abstract}
Nitrogen is the mineral nutrient required most by plants. There are, however, no studies on $\mathrm{N}$ fertilization calibration for rooted cocoa tree cutting production on substrate and frequent irrigation. The objective of this study was to compare the efficiency of urea fertilization on substrate and leaves for rooted cocoa tree cutting and to define $N$ recommendable doses and foliar critical $N$ level. The experiment was set up in a rooted cutting breeding nursery of the Cocoa Biofactory Institute, in Ilheus, Bahia, in July 2006, involving 10 treatments (two fertilization treatments and five urea doses), in random blocks with four repetitions. Each plot consisted of 12 rooted cocoa cuttings in plastic tubes, containing $288 \mathrm{~cm}^{3}$ substrate (50\% coconut fiber and $50 \%$ Plantmax $\left.{ }^{\circledR}\right)$. The substrate was limed with $3.20 \mathrm{~kg} \mathrm{~m}^{-3}$ and fertilized with $2.68 \mathrm{~kg} \mathrm{~m}^{-3}$ of single superphosphate. The treatments were applied between the $82^{\text {nd }}$ and $138^{\text {th }}$ day, with the following weekly $\mathrm{N}$ doses to the substrate $\left(0,20,40,60\right.$ and $\left.80 \mathrm{mg} \mathrm{dm}^{-3}\right)$ and to the leaves $\left(0.0,2.25,4.5,9.0\right.$ and $\left.13.5 \mathrm{~g} \mathrm{~L}^{-1}\right)$. For all treatments, fertilization was supplemented with $P, K, S, Z n$ and $B$ via substrate. On the $145^{\text {th }}$ day, the plants were harvested to evaluate diameter, height, leaf area, shoot dry matter (SDM), nutrient concentration in the diagnostic leaf and nutrient content in the shoot. The biometric and nutrient variables responded to treatments. Adding urea to the substrate led to the maximum yield, exceeding foliar fertilization for SDM, height and leaf area. The $N$ doses recommended to obtain $99 \%$ of the maximum yield varied, according to the biometric variable, from 43 to $76 \mathrm{mg} \mathrm{dm}^{-3}$ for fertilization on substrate and from 9.1 to $11.7 \mathrm{~g} \mathrm{~L}^{-1}$ for foliar fertilization. The critical foliar $N$ level to obtain $99 \%$ of the maximum yield of SDM by foliar fertilization was $23.1 \mathrm{~g} \mathrm{~kg}^{-1} \mathrm{~N}$.
\end{abstract}

Index terms: Theobroma cacao, nutrition, foliar fertilizer, critical level, soilless crop, nitrogen.

\section{INTRODUÇÃO}

Distúrbios nutricionais têm sido observados no sistema de produção de mudas de cacau clonal no Instituto Biofábrica de Cacau (Marrocos \& Sodré, 2004; Souza Jr. et al., 2006); após pesquisas de calibração da calagem (Campos et al., 2006) e de adubação com P nos substratos (Carmello \& Souza Jr., 2006b), o N e o $\mathrm{K}$ foram considerados os nutrientes mais desbalanceados, para o atual sistema (Souza Jr. et al., 2006). O N, além de ser um nutriente que limita bastante o crescimento de mudas de cacau (Morais et al., 1979), pode ser perdido por volatilização e, em ambiente com irrigação constante, sofre perdas elevadas por lixiviação.

A calagem deve ser efetuada no preparo do substrato (Bataglia \& Furlani, 2004; Campos et al., 2006) e o $P$ também pode ser adicionado nesse momento, desde que o substrato tenha capacidade de adsorvê-lo e liberá-lo posteriormente (Williams \& Nelson, 1996; Bataglia \& Furlani, 2004; Carmello \& Souza Jr., 2006b). No entanto, a lixiviação elevada implica que a adubação dos nutrientes de alta mobilidade deve ser realizada em pós-plantio, após o enraizamento da estaca e quando ela começa a emitir ramos novos.

Mudas de cacau são indiferentes à fonte de $\mathrm{N}$, seja ela nítrica, amoniacal ou combinações destas (Santana et al., 1980), e respondem similarmente à aplicação de uréia, quando comparada com o sulfato de amônio, desde que não haja deficiência de S (Miranda \& Morais, 1971).
Para mudas e plantas adultas de cacau, encontraram-se pesquisas utilizando adubação foliar com concentração de uréia variando de 5 a $10 \mathrm{~g} \mathrm{~L}^{-1}$ (Jaramillo, 1952, 1953; Morais et al., 1979; Quintero et al., 1991) a até $50 \mathrm{~g} \mathrm{~L}^{-1}$ (Cain, 1956), sendo a taxa de absorção considerada rápida, pois folhas em diferentes estádios de maturação absorvem em média 60 e $90 \%$ da uréia aplicada após duas horas, pelas faces adaxial e abaxial, respectivamente (Cain, 1956).

Os objetivos deste trabalho foram avaliar a resposta de mudas clonais de cacau a doses e formas de adubação com uréia, comparar a eficiência das adubações no substrato e foliar e definir doses recomendáveis e nível crítico foliar de $\mathrm{N}$.

\section{MATERIAL E MÉTODOS}

O experimento foi instalado em viveiro de produção de mudas, com laterais e teto de tela plástica preta com $50 \%$ de sombreamento, no Instituto Biofábrica de Cacau (IBC), em Ilhéus, Bahia, em julho de 2006.

Para a produção das mudas por propagação vegetativa, foram utilizadas estacas apicais semiherbáceas, de ramos plagiotrópicos de cacaueiros do clone $\mathrm{PH}$ 16, com aproximadamente $18 \mathrm{~cm}$ de comprimento, contendo três folhas, com $1 / 2$ a 2/3 do limbo foliar remanescente. Elas foram tratadas na base com $6 \mathrm{~g} \mathrm{~kg}^{-1}$ de AIB em talco e estaqueadas em tubetes de $288 \mathrm{~cm}^{3}$, contendo substrato; a 
profundidade de estaqueamento foi em torno de $6 \mathrm{~cm}$. Foram preparados 1.512 tubetes, com uma estaca por tubete.

O substrato utilizado foi formado, em volume, por $50 \%$ de fibra de coco (FC) e $50 \%$ de Plantmax ${ }^{\circledR}(\operatorname{Pmax}$ - substrato comercial, recomendado para produção de mudas de espécies florestais propagadas por estaquia, composto de casca de pínus compostada, vermiculita expandida, carvão granulado e turfa). Ambos os substratos foram peneirados $(5 \mathrm{~mm})$ e posteriormente misturados em betoneira, onde receberam calcário e superfosfato simples, ambos peneirados $(0,84 \mathrm{~mm})$, nas doses respectivas de 3,20 e $2,68 \mathrm{~kg} \mathrm{~m}^{-3}$, definidas em experimentos anteriores (Campos et al., 2006; Carmello \& Souza Jr., 2006b).

Utilizou-se irrigação automática do tipo nebulização intermitente, com água do rio Almada (CE 0,088 dS m-1 e pH 7,34), com funcionamento do sistema das 6 às $18 \mathrm{~h}$, sendo este desligado nos períodos com chuva. Utilizaram-se bicos com vazão teórica e efetiva, respectivamente, de 40 e $52 \mathrm{~L} \mathrm{~h}^{-1}$, com área irrigada de $4 \mathrm{~m}^{2}$ por bico. Para a fase de enraizamento, adotou-se um período de 45 dias, em que a irrigação foi efetuada a cada $5 \mathrm{~min}$, durante 25 a $30 \mathrm{~s}$. Até os 95 dias, gradativamente, o intervalo entre irrigações foi sendo reduzido e o tempo de irrigação foi aumentando, até atingir três irrigações diárias, às 7 , 11 e 15 h; nessa fase, o sistema de irrigação passou a ser administrado manualmente. O tempo de irrigação foi ajustado de acordo com a fase de crecimento das mudas e as condições climáticas, até alcançar 10 a 15 min por irrigação.

O controle fitossanitário, num total de sete aplicações durante o período do experimento, foi semelhante ao adotado pelo IBC, com a utilização de fungicidas, acaricidas e inseticidas, à base de mancozeb, endosulfan, methamidophos, deltamethrin e óxido cuproso, com, respectivamente, três, duas, duas, uma e uma aplicações.

A partir da 11a semana, os tratamentos e as parcelas foram identificados, sendo a parcela útil mantida com 12 mudas. Além dessas, mais 43 mudas por tratamento foram identificadas e mantidas como sobressalentes para possível reposição nas parcelas úteis, se necessário.

O experimento foi constituído por 10 tratamentos: duas formas de adubação com uréia (no substrato ou foliar) e cinco doses, em delineamento de blocos ao acaso, com quatro repetições. Os tratamentos foram aplicados semanalmente, sempre após a última irrigação diária, num total de nove adubações, entre o $82^{\circ}$ e o $138^{\circ}$ dia após o estaqueamento.

Quanto à adubação no substrato, as doses semanais de $\mathrm{N}$ foram de 0, 20, 40, 60 e $80 \mathrm{mg} \mathrm{dm}^{-3}$, aplicadas na forma líquida, sendo utilizados $2,0 \mathrm{~mL}$ da solução por tubete; após essa adubação, ligava-se o sistema de irrigação por aproximadamente dois minutos, apenas para auxiliar a penetração da solução no substrato, sem gerar lixiviação.
Para as adubações foliares com soluções de uréia, separaram-se espacialmente as parcelas por tratamentos, para evitar possível contaminação entre estes, e esperava-se a evaporação da água remanescente sobre as folhas, oriunda da última irrigação, para se proceder às aplicações foliares com soluções nas concentrações de $\mathrm{N}$ de 0,$0 ; 2,25 ; 4,5 ; 9,0$; e 13,5 g L-1. Pulverizavam-se as mudas até o início do escorrimento foliar das soluções.

Nos mesmos dias das aplicações dos tratamentos, todas as parcelas também receberam adubação complementar semanal, via substrato, com K, S, Zn e B, nas doses respectivas de $30 ; 12,7 ; 0,5$; e $0,1 \mathrm{mg} \mathrm{dm}^{-3}$, na forma de sulfato de potássio, sulfato de zinco e ácido bórico, sendo utilizado 1,0 $\mathrm{mL}$ da solução por tubete. Na segunda e sexta adubações também se aplicou $\mathrm{P}\left(20 \mathrm{mg} \mathrm{dm}^{-3}\right)$, na forma de fosfato monoamônico.

Aos 145 dias, avaliaram-se: diâmetro da haste principal, com auxílio de paquímetro digital, tomandose duas medidas perpendiculares entre si e à distância de aproximadamente um centímetro do ponto de brotamento; e altura da haste principal, medida com régua. A parte aérea das plantas foi colhida (apenas os ramos emitidos), sendo separada em: folha diagnóstica (terceira folha do primeiro lançamento maduro), definida em experimento anterior (Carmello \& Souza Jr., 2006a), demais folhas e caule. A área foliar foi medida com auxílio de medidor de área foliar.

O material vegetal da parte aérea (folhas e caule) foi lavado (rápida imersão em: água corrente, solução de detergente neutro 0,1 \%, água corrente, solução de $\mathrm{HCl} 3 \%$, água destilada e água destilada novamente). As amostras foram secas em estufa com circulação forçada de ar a $65^{\circ} \mathrm{C}$, por três dias, e posteriormente pesadas para se obter: matéria seca da parte aérea (MSPA), que foi a soma das matérias secas das folhas e do caule. As amostras de folhas e caule foram moídas e analisadas quimicamente, de acordo com Embrapa (1999), visando obter o teor de nutrientes na folha diagnóstica e o conteúdo total de nutrientes na parte aérea das plantas.

Os resultados foram submetidos à análise de variância, sendo o fator quantitativo, doses de $\mathrm{N}$, submetido à análise de regressão, com coeficientes linear e quadrático ou raiz-quadrático; foram aceitos os modelos que apresentaram todos os coeficientes significativos a até $10 \%$ pelo teste $\mathrm{F}$ e o maior coeficiente de determinação ajustado. As doses recomendáveis de $\mathrm{N}$ foram calculadas para obter $99 \%$ da produção máxima das variáveis biométricas e, a partir da dose recomendável para MSPA, determinouse o nível crítico foliar.

Os valores máximos estimados das variáveis biométricas para obtenção da produção máxima de MSPA foram analisados por contraste, entre as formas de adubação (substrato e foliar), sendo os contrastes considerados significativos a até $5 \%$ pelo teste F. 


\section{RESULTADOS E DISCUSSÃO}

As mudas de cacau responderam à adição de $\mathrm{N}$, com relação às variáveis biométricas avaliadas, apresentando pontos de produção máxima, visto que houve efeitos linear e quadrático ou raiz-quadrático significativos (Figura 1). Independentemente da forma de adubação, as mudas apresentaram comportamento raiz-quadrático para as variáveis altura e diâmetro (Figura 1c,d,e,f), evidenciando taxa de resposta inicial maior em relação à MSPA que apresentou comportamento quadrático (Figura 1a,b), o que confere resposta inicial menor.

Quanto à adubação foliar, as doses semanais para obtenção da produção máxima variaram de 10,9 a $13,5 \mathrm{~g} \mathrm{~L}^{-1}$ de $\mathrm{N}$ ou 24 a $30 \mathrm{~g} \mathrm{~L}^{-1}$ de uréia (Quadro 1). Essas doses são superiores às utilizadas por Jaramillo (1952, 1953), Morais et al. (1979) e Quintero et al. (1991), de 5 a $10 \mathrm{~g} \mathrm{~L}^{-1}$ de uréia. Vale ressaltar que se observou queima (necrose) das bordas e, principalmente, do ápice foliar, para a dose de $13,5 \mathrm{~g} \mathrm{~L}^{-1} \mathrm{de} \mathrm{N}$.
Em se tratando da adubação no substrato, as doses semanais para obtenção da produção máxima variaram de acordo com a característica considerada: de 63 a $80 \mathrm{mg} \mathrm{dm}^{-3}$ de N (Quadro 1). Essas doses são inferiores à média semanal, relatada por Decarlos Neto et al. (2002), para obtenção de altura máxima de portaenxertos de citros em tubetes aos 120 dias, que foi de $152 \mathrm{mg} \mathrm{dm}^{-3}$ de $\mathrm{N}$, para tubete pequeno com $40 \mathrm{~cm}^{3}$ de substrato. Por sua vez, Esposti \& Siqueira (2004), também estudando a adubação de porta-enxertos de citros, porém cultivados por 305 dias e em tubete grande, contendo $3,8 \mathrm{dm}^{3}$ de substrato, chegaram à dose média quinzenal de $29 \mathrm{mg} \mathrm{dm}^{-3}$ de $\mathrm{N}$, para obtenção da produção máxima para diâmetro, MSPA, altura e área foliar.

As doses recomendáveis, calculadas para obtenção de 99 \% da produção máxima, também variaram de acordo com a variável biométrica considerada, sendo a amplitude de 43 a $76 \mathrm{mg} \mathrm{dm}^{-3}$ de $\mathrm{N}$ para adubação no substrato e de 9,1 a $11,7 \mathrm{~g} \mathrm{~L}^{-1}$ de $\mathrm{N}\left(20\right.$ a $26 \mathrm{~g} \mathrm{~L}^{-1}$ de uréia) para adubação foliar (Quadro 1).

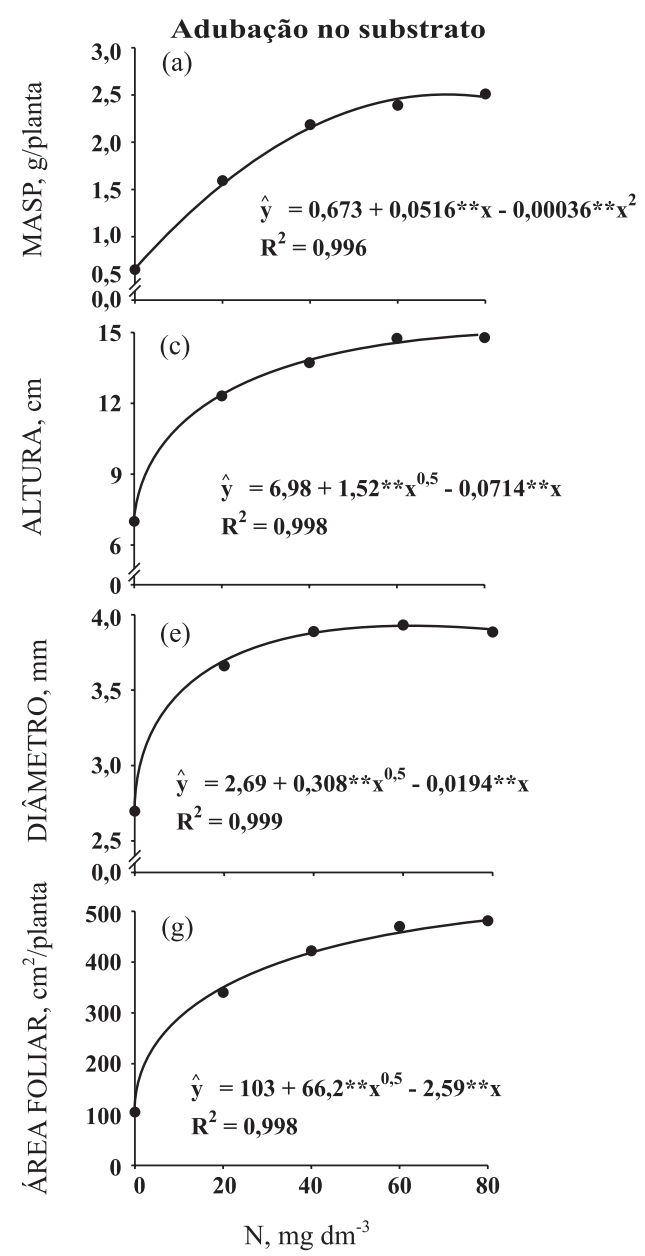

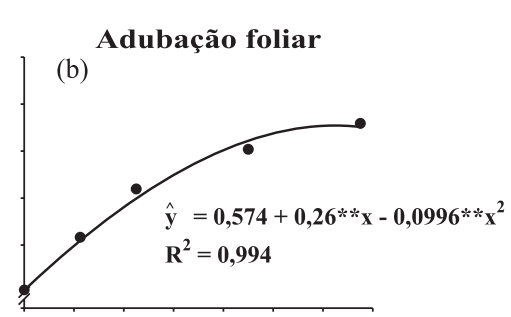

(d)
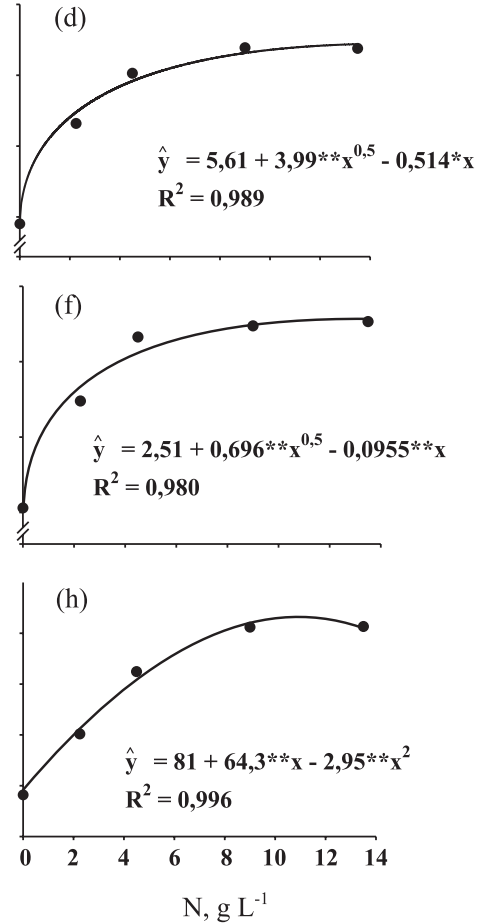

Figura 1. Matéria seca da parte aérea - MSPA, altura, diâmetro e área foliar de mudas de cacaueiro, aos 145 dias, em função da dose de nitrogênio e forma de adubação: no substrato ou foliar. **, significativo a $1 \%$ pelo teste $\mathrm{F}$. 
Independentemente da forma de adubação, o diâmetro do caule propiciou as menores doses recomendáveis (Quadro 1), certamente porque foi a variável que menos discriminou os tratamentos das doses maiores de $\mathrm{N}$ (Figura 1) - tendência semelhante a esta pode ser observada no trabalho de Esposti \& Siqueira (2004), para porta-enxertos de citros. As doses recomendáveis maiores foram obtidas para área foliar em adubação no substrato e para MSPA em adubação foliar (Quadro 1).

A adubação com uréia, independentemente de sua forma de aplicação, aumentou o teor de $\mathrm{N}$ na folha, reduziu o teor de $\mathrm{K}$ e de $\mathrm{Zn}$ e mostrou efeito tipicamente quadrático para $\mathrm{P}$ (Figura 2). O efeito de diluição explica a redução nos teores de $\mathrm{K}$ e de $\mathrm{Zn}$, nutrientes que inclusive foram fornecidos semanalmente pós-plantio, mas cujos teores foliares nos tratamentos com maiores doses de $\mathrm{N}$ ficaram abaixo daqueles considerados adequados para cacaueiros adultos por Malavolta (2006) e próximos ao limite inferior da faixa considerada adequada por Raij et al. (1997), sugerindo uma possível deficiência desses nutrientes. O efeito de diluição também explica a redução inicial do teor de $\mathrm{P}$ com o aumento das doses de N (Figura 2a,b), porém a adubação nitrogenada pode ter favorecido o crescimento do sistema radicular, o que estimularia a absorção de $\mathrm{P}$ e, conseqüentemente, o aumento de seu teor foliar.

Quadro 1. Doses semanais de nitrogênio para obtenção da produção máxima e doses recomendáveis para obter 99 \% da produção máxima para matéria seca da parte aérea (MSPA), altura, diâmetro e área foliar (AF) de mudas de cacau e nível crítico foliar (NCF - para obtenção de 99 \% da produção máxima de MSPA), de acordo com a forma de adubação, aos 145 dias

\begin{tabular}{|c|c|c|c|c|c|c|c|c|c|}
\hline \multirow{2}{*}{$\begin{array}{l}\text { Forma de } \\
\text { adubação }\end{array}$} & \multicolumn{4}{|c|}{ Dose de $\mathrm{N}$ para produção máxima } & \multicolumn{4}{|c|}{ Dose recomendável de $\mathrm{N}$} & \multirow{2}{*}{ NCF } \\
\hline & MSPA & Altura & Diâmetro & AF & MSPA & Altura & Diâmetro & AF & \\
\hline & \multicolumn{8}{|c|}{ - $\mathrm{mg} \mathrm{dm}^{-3}$ (substrato) e g L $\mathrm{L}^{-1}$ (foliar) } & $\mathrm{g} \mathrm{kg}^{-1}$ \\
\hline Substrato & 71 & 80 & 63 & 80 & 63 & 71 & 43 & 76 & 22,9 \\
\hline Foliar & 13,2 & 13,5 & 13,3 & 10,9 & 11,7 & 11,1 & 9,1 & 9,7 & 23,3 \\
\hline
\end{tabular}
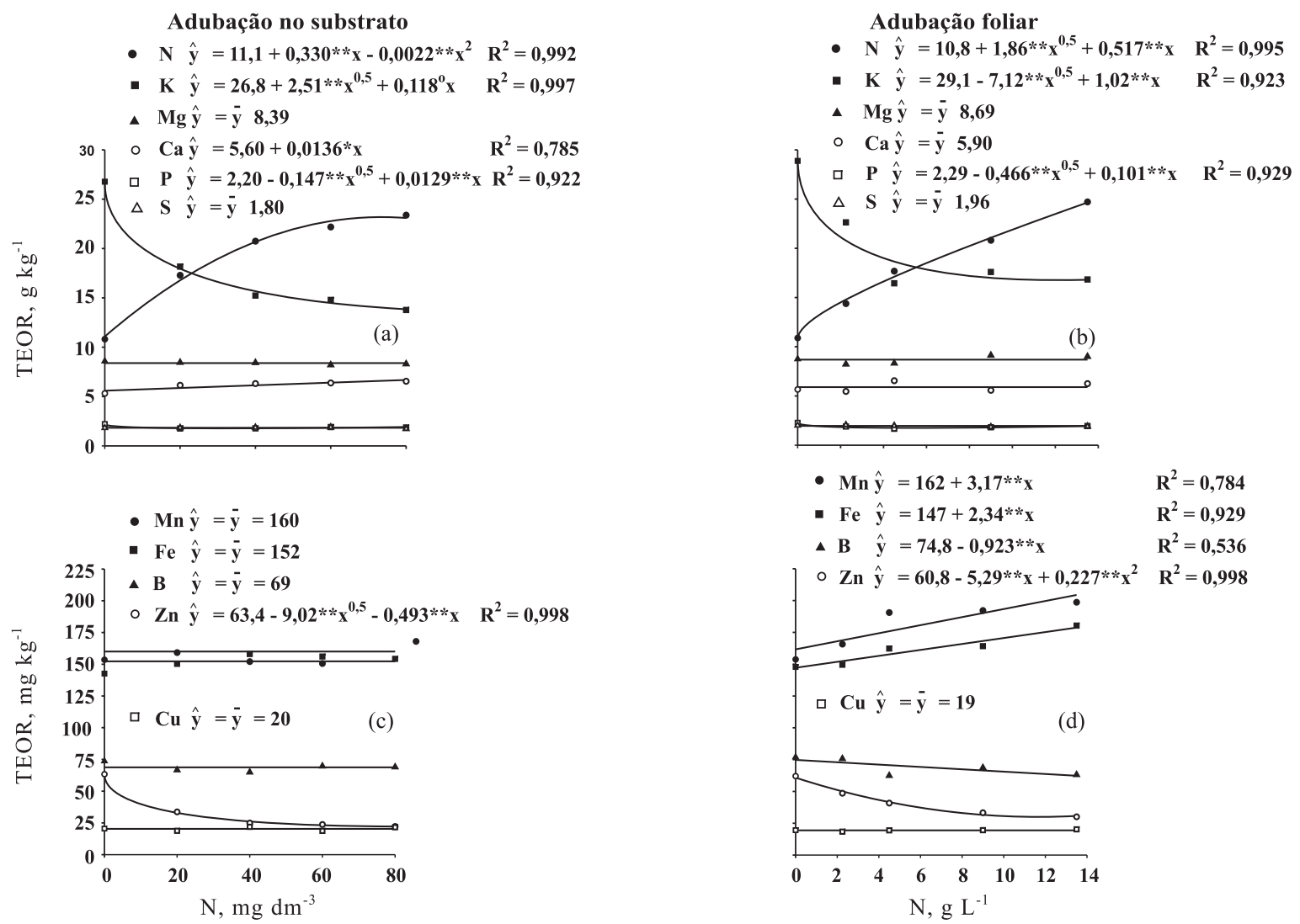

Figura 2. Teor de macro e micronutrientes na folha diagnóstica de mudas de cacaueiro, aos 145 dias, em função da dose de nitrogênio e forma de adubação: no substrato ou foliar. $* *, * e^{\circ}$, significativo a 1,5 e 10 $\%$ pelo teste $\mathrm{F}$, respectivamente. 
A adubação com uréia no substrato aumentou também o teor foliar de Ca, porém não alterou o teor dos demais nutrientes (Figuras 2a,c). Ruschel et al. (2004) também observaram que a adubação nitrogenada no substrato alterou as concentrações de Ca em folhas de mudas de limoeiro, apresentando resposta quadrática. Segundo Basso (1995), as fontes de $\mathrm{N}$ podem alterar a nutrição de $\mathrm{Ca}$ devido a: alteração do $\mathrm{pH}$ externo; competição com $\mathrm{NH}_{4}{ }^{+}$ou estimulação pelo $\mathrm{NO}_{3}{ }^{-}$na absorção de $\mathrm{Ca}$; melhoria do transporte de Ca no xilema, pois o $\mathrm{NO}_{3}{ }^{-}$é o ânion acompanhante preferencial no transporte de Ca dentro da planta.

A adubação foliar com uréia não alterou o teor na folha de $\mathrm{Ca}, \mathrm{Mg}$, $\mathrm{S}$ e $\mathrm{Cu}$, mas reduziu o teor de $\mathrm{B}$ e aumentou a de Fe e Mn (Figura 2).
Independentemente da forma de adubação, o aumento da dose de uréia favoreceu o acúmulo de todos os nutrientes na parte aérea das mudas de cacau (Figura 3), devido ao incremento do teor de alguns nutrientes (Figura 2) e, principalmente, ao aumento da matéria seca (Figura 1a,b). Para a adubação no substrato, a variação do incremento no conteúdo de nutrientes foi: $\mathrm{N}>\mathrm{Mn}>\mathrm{P}>\mathrm{Fe} \cong \mathrm{S} \cong \mathrm{B} \cong \mathrm{Mg} \cong \mathrm{Ca}>$ $\mathrm{K}>\mathrm{Zn}>\mathrm{Cu}$. Essa seqüência foi semelhante à observada para a adubação foliar: $\mathrm{N}>\mathrm{Fe} \cong \mathrm{P} \cong$ $\mathrm{Mn} \cong \mathrm{S} \cong \mathrm{Mg} \cong \mathrm{Ca} \cong \mathrm{B}>\mathrm{K}>\mathrm{Zn}>\mathrm{Cu}$.

A análise por contraste das produções máximas estimadas dentro do espaço experimental, entre as duas formas de fornecimento de uréia, apontou que a adubação no substrato foi superior à foliar para MSPA, altura e área foliar (Quadro 2), com incrementos
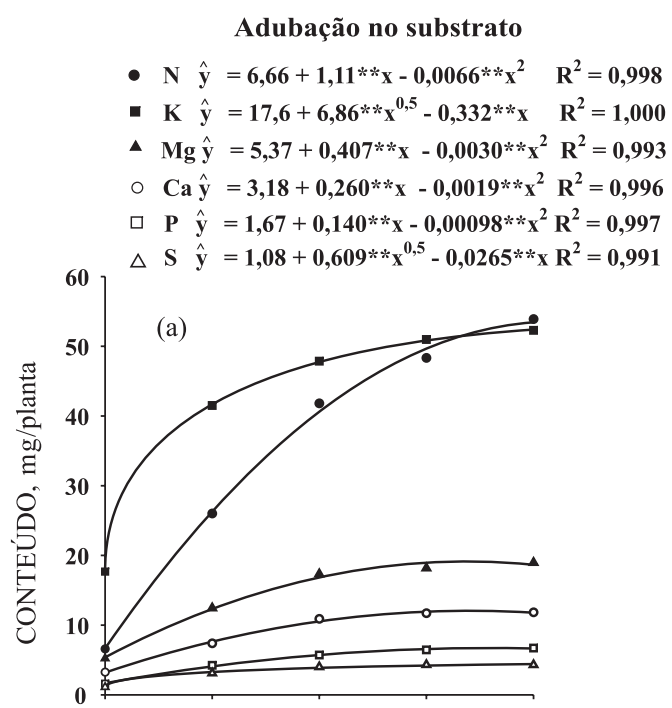

- Mn $\hat{\mathbf{y}}=104+3,86^{* *} \mathrm{x}$

$\mathrm{Fe} \hat{\mathbf{y}}=91,2+7,61 * * \mathrm{x}-0,0538 * * \mathrm{x}^{2} \quad \mathrm{R}^{2}=0,971$

B $\quad \hat{\mathbf{y}}=37,6+3,13 * * \mathrm{x}-0,0237 * * \mathrm{x}^{2} \quad \mathrm{R}^{2}=0,993$

$Z n \quad \hat{y}=38,2+13,8 * * x^{0,5}-0,852 * * x \quad R^{2}=0,981$

ㄷ Cu $\hat{y}=20,7+0,96 * * x-0,0083 * * x^{2} \quad R^{2}=0,990$

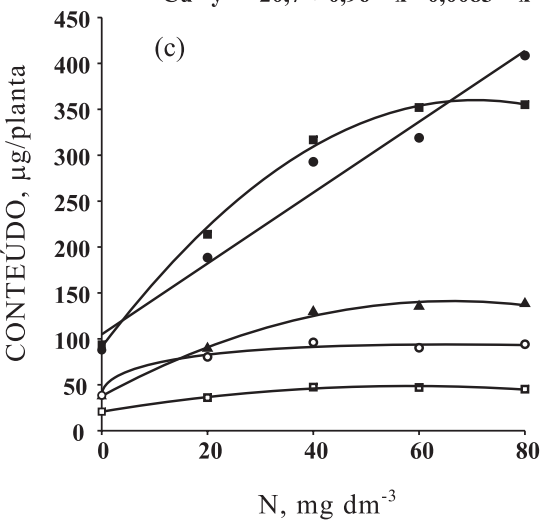

Adubação foliar

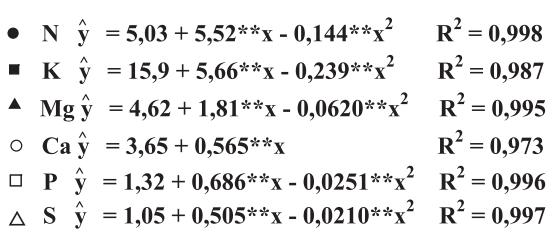$$
\text { (b) }
$$
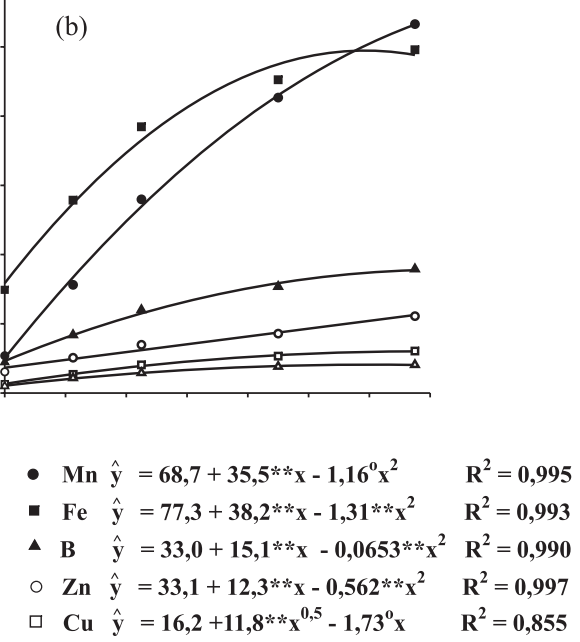

(d)

(d)

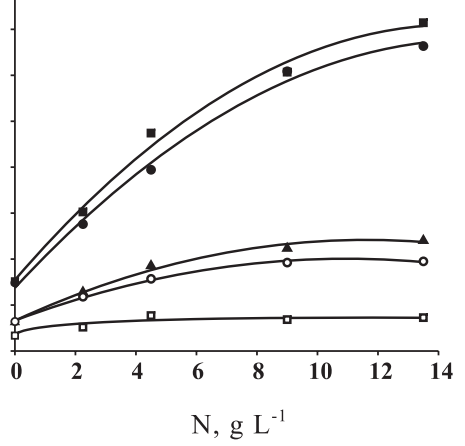

Figura 3. Conteúdo de macro e micronutrientes na parte aérea de mudas de cacaueiro, aos 145 dias, em função da dose de nitrogênio e forma de adubação: no substrato ou foliar. ** e *, significativo a 1 e $5 \%$ pelo teste $\mathrm{F}$, respectivamente. 
Quadro 2. Contrastes dos valores estimados para produção máxima da matéria seca da parte aérea (MSPA), altura, diâmetro da haste e área foliar de mudas de cacau, de acordo com a forma de adubação, aos 145 dias

\begin{tabular}{lccc}
\hline Forma de adubação & MSPA & Altura & Diâmetro \\
\hline & g/planta & $\mathrm{cm}$ & $\mathrm{mm}^{2} / \mathrm{planta}$ \\
Substrato & 2,51 & 14,9 & 3,92 \\
Foliar $_{\text {Contraste }}^{(1)}$ & 2,27 & 13,3 & 3,78 \\
\hline
\end{tabular}

${ }^{(1)}$ Diferença entre a adubação via substrato e foliar; ** $\mathrm{e} *$ significativo a 1 e $5 \%$ pelo teste $\mathrm{F}$, respectivamente.

respectivos de 10, 12 e $13 \%$. Contudo, vale ressaltar que a adubação foliar seria mais fácil de ser implementada em um sistema massal de produção de mudas, desde que houvesse o fornecimento dos outros nutrientes não supridos satisfatoriamente pelo substrato e o aumento da freqüência de adubação.

Os teores estimados de $\mathrm{N}$ na folha diagnóstica para obtenção da produção máxima da MSPA não diferiram significativamente e, conseqüentemente, os níveis críticos foliares de N, calculados para obtenção de $99 \%$ da produção máxima da MSPA, foram semelhantes entre as duas formas de adubação (Quadro 1), podendose admitir um teor médio de $23,1 \mathrm{mg} \mathrm{kg}^{-1}$ de $\mathrm{N}$, valor que é superior aos $20 \mathrm{~g} \mathrm{~kg}^{-1}$ apontados por Malavolta (2006) como adequado para cacaueiros adultos, estando porém dentro da faixa de suficiência de 20 a $25 \mathrm{~g} \mathrm{~kg}^{-1}$ sugerida por Raij et al. (1997) também para cacaueiros. Para mudas de cacau cultivadas em solução nutritiva, Loué (1962) concluiu que havia deficiência média de $\mathrm{N}$ quando o teor foliar se encontrava entre 20 e $22 \mathrm{~g} \mathrm{~kg}^{-1}$ e ligeira quando o teor era de $23 \mathrm{~g} \mathrm{~kg}^{-1}$ de $\mathrm{N}$.

\section{CONCLUSÕES}

1. As mudas de cacau responderam às formas e doses de adubação com uréia.

2. A adubação no substrato foi superior à adubação foliar, para aplicações semanais.

3. As doses recomendáveis de $\mathrm{N}$ variaram de 43 a $76 \mathrm{mg} \mathrm{dm}^{-3}$ para adubação no substrato e de 9,1 a $11,7 \mathrm{~g} \mathrm{~L}^{-1}$ para adubação foliar.

4. O nível crítico foliar de $\mathrm{N}$ foi de $23,1 \mathrm{~g} \mathrm{~kg}^{-1}$.

\section{LITERATURA CITADA}

BASSO, C. Influence of potassium, calcium, and magnesium availability in the soil on nutrients in leaves and fruits, and the evolution of surface wax and nutrients during apple fruit development. Raleigh, North Carolina State University, 1995. 109p. (Tese de Doutorado)
BATAGLIA, O.C. \& FURLANI, P.R. Nutrição mineral e adubação para cultivos em substratos com atividade química. In: ENCONTRO NACIONAL SOBRE SUBSTRATOS PARA PLANTAS, 4., Viçosa, 2004. Anais. Viçosa, MG, Universidade Federal de Viçosa, 2004. p.106128.

CAIN, J.C. Absorption and metabolism of urea by leaves of coffee, cacao and banana. Proc. Am. Soc. Hortic. Sci., 67:279-286, 1956.

CAMPOS, V.P.; GATTWARD, J.N.; SOUZA JR., J.O.; MENEZES, A.A.; PAIVA, A.Q.; SACRAMENTO, C.K.; ALMEIDA, A.A.F. \& FARIA, J.C.F. Biomassa e $\mathrm{pH}$ do substrato em função do calcário e do substrato na propagação vegetativa do cacaueiro. In: ENCONTRO NACIONAL SOBRE SUBSTRATOS PARA PLANTAS, 5., Ilhéus, 2006. Anais. Ilhéus, Comissão Executiva do Plano da Lavoura Cacaueira, 2006. p.158.

CARMELLO, Q.A.C. \& SOUZA JR., J.O. Metodologias utilizadas para avaliação da fertilidade de solos podem ser aplicadas a substratos? I. Macronutrientes. In: ENCONTRO NACIONAL SOBRE SUBSTRATOS PARA PLANTAS, 5., Ilhéus, 2006. Anais. Ilhéus, Comissão Executiva do Plano da Lavoura Cacaueira, 2006a. p.103.

CARMELLO, Q.A.C. \& SOUZA JR., J.O. Sobrevivência e crescimento de mudas clonais de cacau em função dos substratos e das doses e época de aplicação de P. In: INTERNATIONAL COCOA RESEARCH CONFERENCE, 15., San José, 2006. Proceedings. San José, Cocoa Producers' Alliance, 2006b. p.179.

DECARLOS NETO, A.; SIQUEIRA, D.L.; PEREIRA, P.R.G. \& ALVAREZ V., V.H. Crescimento de porta-enxertos de citros em tubetes influenciados por doses de N. R. Bras. Frutic., 24:199-203, 2002.

EMPRESA BRASILEIRA DE PESQUISA AGROPECUÁRIA EMBRAPA. Manual de análises químicas de solos, plantas e fertilizantes. Brasília, 1999. 370p.

ESPOSTI, M.D.D. \& SIQUEIRA, D.L. Doses de uréia no crescimento de porta-enxertos de citros produzidos em recipientes. R. Bras. Frutic., 26:136-139, 2004.

JARAMILLO A., R. La urea y el marchitamiento de frutos jóvenes: Nota preliminar. Cacao en Colombia, 1:101-106, 1952. 
JARAMILLO, A.R. La urea y el marchitamiento de los frutos jóvenes de cacao. Cacao en Colombia, 2:63-76, 1953.

LOUÉ, A. Estudo das carências minerais do cacau por intermédio de ensaios em meio artificial. Fertilité, 14:541, 1962.

MALAVOLTA, E. Manual de nutrição mineral de plantas. São Paulo, Ceres, 2006. 631p.

MARROCOS, P.C. \& SODRÉ, G.A. Sistema de produção de mudas de cacaueiros. In: ENCONTRO NACIONAL SOBRE SUBSTRATO PARA PLANTAS, 4., 2004, Viçosa, MG. Anais. Viçosa, MG, Universidade Federal de Viçosa, 2004. p.283-311.

MIRANDA, E.R. \& MORAIS, F.I.O. Efeitos da combinação de diferentes fontes de nitrogênio e potássio no desenvolvimento de plântulas de cacau. R. Theobroma, 1:29-38, 1971.

MORAIS, F.I., SILVA, L.F.; MARINHO, A.H. \& PINHO, A.F.S Efeitos da adubação foliar e de substratos no crescimento de plântulas de cacau. R. Theobroma, 9:163-171, 1979.

QUINTERO, E.; ISAAC, E.; PIERRE, M. \& AGUILAR, P. Aplicación de urea en plantas obtenidas por estacas de Theobroma cacao en la fase de vivero y endurecimiento. R. Baracoa, 21:34-37, 1991.
RAIJ, B.van; CANTARELLA, H. \& QUAGGIO, J.A. Estimulantes. In: RAIJ, B.van; CANTARELLA, H.; QUAGGIO, J.A. \& FURLANI, A.M.C., eds. Recomendação de adubação e calagem para o Estado de São Paulo. 2.ed. Campinas, Instituto Agronômico, 1997. p.93-95. (Boletim Técnico, 100)

RUSCHEL, J.; CARMELLO, Q.A.C.; BERNARDI, A.C.C.; CARVALHO, S.A. \& MATTOS JR., D. Leaf nutrient contents of rangpur lime rootstock as afected by N, P, K, $\mathrm{Ca}$ and S fertilization. Sci. Agric., 61:501-506, 2004.

SANTANA, M.B.M.; EZETA, F.N. \& MORAIS, F.I. Efeito de formas de nitrogênio no crescimento e na composição química de plântulas de cacau. R. Theobroma, 10:31-39, 1980.

SOUZA JR., J.O.; RAMOS, A.; BRITO, A.M.L.; SODRÉ, G.A. \& CARMELLO, Q.A.C. Diagnóstico nutricional de mudas clonais de cacau produzidas pelo Instituto Biofábrica de cacau (IBC), em Ilhéus, Bahia, no ano de 2005. In: ENCONTRO NACIONAL SOBRE SUBSTRATOS PARA PLANTAS, 5., Ilhéus, 2006. Anais. Ilhéus, Comissão Executiva do Plano da Lavoura Cacaueira, 2006. p.160.

WILLIAMS, K.A. \& NELSON, P.V. Modifying a soilless root medium with aluminum influences phosphorus retention and Chrysanthemum growth. HortScience, 31:381-384, 1996. 